\title{
COLABORAÇÃO INTERPROFISSIONAL EM EQUIPE: PERCEPÇÃO DE PROFISSIONAIS DE SAÚDE DA REGIÃO NORDESTE DO BRASIL ${ }^{1}$
}

\author{
INTERPROFESSIONAL TEAM COLLABORATION: HEALTH \\ PROFESSIONALS' PERCEPTIONS IN NORTHEASTERN BRAZIL
}

\author{
Emanuella Pinheiro de Farias Bispo ${ }^{2}$ \\ Rosana Aparecida Salvador Rossit ${ }^{3}$ \\ Carole Orchard ${ }^{4}$
}

\begin{abstract}
RESUMO: A Educação Interprofissional (EIP) é uma estratégia inovadora que proporciona a prática colaborativa que ocorre quando duas ou mais profissões aprendem sobre os outros, com os outros e entre si. Com o objetivo de analisar a percepção de profissionais do Nordeste do Brasil em relação à colaboração interprofissional nas equipes de saúde, 245 profissionais de 13 profissões da saúde completaram a AITCS II-BR que avalia o nível de colaboração interprofissional entre os membros de uma equipe. A escala possui 23 assertivas organizadas em três dimensões e foi hospedada em plataforma online. Os dados receberam tratamento estatístico e as respostas foram classificadas em zona de conforto, alerta e perigo. Identificou-se fragilidades relacionadas à parceria, cooperação e coordenação nas equipes. A colaboração interprofissional ainda é tema pouco explorado no contexto da formação inicial e permanente dos profissionais de saúde.
\end{abstract}

Palavras chave. Educação interprofissionais. Aprendizado colaborativo. Formação. Equipe de assistência ao paciente.

\footnotetext{
1 Este capítulo é parte adaptada da tese de doutoramento "Tradução e a adaptação transcultural do Assessment of Interprofessional Team Collaboration Scale II - AITCS II para profissionais da área de saúde destinada à realidade brasileira" desenvolvida no Programa de Pós-Graduação Interdisciplinar em Ciências da Saúde, UNIFESP-BS e apresenta o estudo referente à tradução, adaptação transcultural e validação do Assessment of Interprofessional Team CollaborationScale II (AITCS II, ORCHARD, 2015) para o português brasileiro.

2 Terapeuta Ocupacional, Professora Doutora da Universidade Estadual de Ciências da Saúde de Alagoas (UNCISAL). Endereço: Rua Prefeito Abdon Arroxelas, oㅡ 605, apto.304. Ponta Verde. CEP.: 57-035.380. Maceió-AL. E-mail: emanuellapinheirofbispo@gmail.com.

${ }^{3}$ Terapeuta Ocupacional, Professora Doutora Visitante Universidade Federal de São Paulo-CEDESS

${ }^{4}$ Médica, Professora Doutora da University of Western Ontario - Canadá.
} 
ABSTRACT: Interprofessional Education (IPE) is an innovative strategy focused on enabling the collaborative practice that takes place when two, or more, professions learn about others, from others, with each other. The aim of the current study is to analyze the perception of 245 professionals from 13 health-related fields about interprofessional collaboration among health teams in Northeastern Brazil. These professionals completed the Assessment of Interprofessional Team Collaboration Scale II (AITCS II-BR), which assesses the interprofessional collaboration level among team members. The scale, which comprises 23 assertions organized in three dimensions, was hosted on an online platform. Data were subjected to statistical treatment and responses were categorized into comfort, risk and danger zones. Partnership, cooperation and coordination weaknesses were identified in the assessed teams. Interprofessional collaboration remains little explored in initial and permanent health professional training processes.

Keywords. Interprofessional education. Collaborative learning. Training. Patient care team. 


\section{INTRODUÇÃO}

A Organização Mundial da Saúde (OMS, 2010) reconhece que a Educação Interprofissional (EIP) é uma estratégia inovadora que proporciona a prática colaborativa que desempenhará um papel importante na redução da crise mundial na força de trabalho em saúde.

A colaboração interprofissional é um tipo de trabalho interprofissional que envolve diferentes profissões da saúde ou da assistência social e que se reúnem regularmente para prestar assistência. A colaboração compreende o compartilhamento das responsabilidades, parceria, interdependência entre os indivíduos, clareza das funções e dos objetivos, poder e envolve a reflexão e ação mútua em busca de respostas às necessidades de saúde dos usuários (Reeves, Xyrichis, Zwarenstein, 2018; Reeves et al., 2010; Barr et al., 2005).

As competências para a prática colaborativa interprofissional envolvem um conjunto integrado de conhecimentos, habilidades, valores e atitudes que possibilitam o trabalho conjunto entre profissões e com pacientes, em associação com as famílias e comunidades, no sentido de melhorar os resultados de saúde (Interprofessional Educational Collaborative, 2016; Khalili, Gilbert, Lising, MacMillan, Maxwell e Xyrichis, 2019).

O documento Core competencies for interprofessional collaborative practice (IPEC, 2016) lista quatro competências fundamentais para o trabalho interprofissional: ética; responsabilidade; comunicação interprofissional; e, trabalho em equipe. Estas competências servem para preparar os estudantes e profissionais para o trabalho colaborativo em equipes de saúde (Thibault, 2013).

Para o trabalho em equipe interprofissional considera-se os níveis de parceria, cooperação e coordenação. Estas dimensões são avaliadas na AITCS IIBR (BISPO; ROSSIT, 2018) e caracterizam as relações entre profissões na prestação de cuidados centrados no paciente/usuário (HPAC, 2019). 
O estudante/profissional esta apto para a prática colaborativa quando sentese preparado e demonstra essa competência e confiança ao trabalhar colaborativamente dentro de uma equipe interprofissional para melhorar a qualidade da atenção e/ou para estabelecer vínculos com os quatro objetivos: melhor atenção, melhor saúde, melhor valor e a melhor experiência de trabalho (Bodenheimer, Sinsky, 2014; Feeley, 2017; Khalili et al., 2019; Organização Mundial da Saúde, 2010).

Apesar dessas iniciativas, a ênfase na formação dos profissionais da saúde continua, predominantemente, na perspectiva da educação uniprofissional, na qual os estudantes de diferentes áreas são formados isoladamente de outras profissões correlatas (Frenk et al., 2010; Khalili, Hall, \& Deluca, 2014; Price, Doucet, \& Hall, 2014).

Dessa forma, a EIP propõe mudanças nos níveis educacionais, profissionais e organizacionais, possibilitando aos estudantes e profissionais a oportunidade de uma formação focada em uma aprendizagem que potencializa desenvolver habilidades e competências colaborativas nas atividades integradas em saúde (REEVES, 2013, 2016).

Os princípios da EIP se aplicam tanto para a formação inicial quanto para a educação permanente dos profissionais da saúde (Barr, 2005), para desenvolver o trabalho colaborativo em saúde, que é fundamental para a integralidade do cuidado (Rossit et al., 2014).

A OMS aponta que a EIP é um passo importante da força de trabalho de saúde para que esteja mais bem preparada para responder às necessidades de saúde. Um profissional de saúde "colaborativo preparado para a prática" é aquele que aprendeu como trabalhar em uma equipe interprofissional e tem competência para este fim (OMS, 2010, p.7).

Para a construção de um ambiente favorável para 0 aprendizado interprofissional é necessário desenvolver estudos, no Brasil, que permitam conhecer que tipo de educação funciona melhor, em que circunstâncias e como esta pode impactar nos resultados da atenção à saúde de usuários e população, trabalhadores e organizações (Peduzzi, 2015). 
Orchard et al. (2012) afirmam ser necessária a existência de instrumentos que permitam avaliar as relações colaborativas em/nas equipes. A escala AITCS II (ORCHARD, 2015) é um instrumento diagnóstico canadense, projetado para medir a colaboração interprofissional entre os membros da equipe. Recentemente, a Escala de Avaliação da Colaboração Interprofissional em Equipe - AITCS II-BR (BISPO e ROSSIT, 2018) passou por processo de tradução, adaptação transcultural e validação para o contexto brasileiro.

Assim, o presente estudo foi desenvolvido para analisar a percepção de profissionais de saúde do Nordeste do Brasil em relação à colaboração interprofissional nas equipes de saúde.

\section{MÉTODO}

Este estudo possibilitou a análise dos níveis de colaboração entre os membros de uma equipe, a partir da percepção dos profissionais de saúde inseridos em cenários de atenção à saúde na região Nordeste do Brasil que participaram da pesquisa e responderam a escala AITCS II-BR (BISPO e ROSSIT, 2018).

A pesquisa foi registrada na Plataforma Brasil e aprovada pelo Comitê de Ética em Pesquisa da Universidade Federal de São Paulo seguindo os preceitos da Resolução 466/12 de 12 de dezembro de 2012, com o Protocolo de aprovação CAAE: 55262416.5.0000.5505, Parecer ํㅜ 3.165.155 de 05 de Julho de 2016.

O cenário da pesquisa corresponde aos serviços de saúde do setor público do Nordeste do Brasil em que os profissionais trabalham com profissionais de diferentes áreas de formação: Atenção Básica, incluindo a Estratégia de Saúde da Família (ESF) e as equipes dos Núcleos de Apoio à Saúde da Família e Atenção Básica (NASF-AB); Equipes dos Centros de Atenção Psicossocial (CAPS) e equipes de Saúde do Trabalhador, além de contar com a participação de profissionais de saúde que exercem, concomitantemente, a atuação nos cenários da assistência e da docência em cursos de graduação da área da saúde. 
Para a composição da amostra foram realizadas buscas nos sites oficiais das Secretarias Estaduais de Saúde dos nove estados da região e das Secretarias Municipais de Saúde das capitais dos estados da região Nordeste com o intuito de obter endereços eletrônicos destes órgãos para viabilizar a coleta de dados.

Como critério de inclusão foram considerados os profissionais que atuavam em cenários de prática junto a diferentes categorias na área da saúde do Nordeste do Brasil. Não participaram os profissionais que não concordaram com os termos da pesquisa, não quiseram participar ou que não estavam disponíveis para participar durante o período definido para a coleta de dados.

Cerca de 1.000 contatos foram fornecidos pelas Secretarias Estaduais e Municipais de Saúde, além de contatos diretos com os cursos de graduação na saúde e com profissionais dos serviços de saúde.

Tendo obtido os contatos, uma carta convite contendo esclarecimentos sobre a pesquisa, a solicitação de encaminhamento aos profissionais vinculados aos serviços de saúde e o link de acesso ao formulário eletrônico. Esta carta foi encaminhada por correio eletrônico às Secretarias Estaduais e Municipais de Saúde, com a finalidade de solicitar a divulgação da pesquisa e sensibilizar os profissionais/serviços para participarem. Ao acionar o link de acesso ao formulário eletrônico, o TCLE era apresentado ao participante e mediante a concordância com os termos da pesquisa, a escala AITCS II-BR era disponibilizada para respostas.

De aproximadamente 1.000 convites, presencial ou por correio eletrônico, participaram efetivamente 245 profissionais de 13 profissões da saúde do Nordeste brasileiro: Serviço Social, Cirurgião-dentista, Enfermagem, Farmácia, Fonoaudiologia, Fisioterapia, Medicina, Nutrição, Psicologia, Terapia ocupacional, Agente comunitário de saúde, Auxiliar em saúde bucal, Técnico de Enfermagem.

Como instrumento de coleta de dados foi utilizado a versão validada da Escala de Avaliação da Colaboração Interprofissional em Equipe - AITCS II-BR (APÊNDICE 1).

A AITCS II-BR (BISPO e ROSSIT, 2018) é um instrumento diagnóstico adaptado e validado para o português brasileiro a partir da Assessment of Interprofessional Team Collaboration Scale II - AITCS II (ORCHARD, 2015), desenvolvida para medir a colaboração interprofissional entre os membros de uma 
equipe. Possui 23 assertivas consideradas características da colaboração interprofissional, entendida a partir de como uma equipe trabalha e atua. Para a aplicação da AITCS II-BR, nas versões impressa e online, as assertivas foram, intencionalmente quase randomizadas, tendo o objetivo de mesclar os itens das dimensões para evitar que o participante ficasse sob o controle de uma variável específica, o que poderia produzir vieses nas respostas.

Os itens da escala AITCS II-BR estão organizados em três dimensões (Parceria, Cooperação e Coordenação) consideradas fundamentais para a prática colaborativa. A partir de uma frase de contextualização "Quando estamos trabalhando em equipe, os profissionais da minha equipe..." existente na escala, todas as 23 assertivas complementam esta frase. Frente aos conteúdos abordados em cada item, o participante assinala o nível de concordância ou discordância. Os respondentes poderiam escolher entre cinco opções: 1="Nunca"; 2="Raramente"; 3="Às vezes"; 4="Frequentemente"; e, 5="Sempre".

O tempo para completar o instrumento foi de aproximadamente 15 minutos.

Para os profissionais que completaram a escala na modalidade impressa, os dados foram inseridos (pela pesquisadora) na plataforma Google Forms para constituir um único banco de dados. O banco de dados em planilha de Excel ${ }^{\circledR}$, gerado automaticamente no Google Forms, foi preparado para possibilitar a análise estatística e análise de conteúdo.

Para o processamento das análises estatísticas, foi utilizado o software IBM SPSS Statistics version 17. As respostas foram submetidas ao processo de validação de conteúdo que contemplou o cálculo do coeficiente de correlação linear (r) e a confiabilidade do instrumento.

Foram consideradas as médias dos itens da escala, sendo que diante de cada item, a concordância plena foi pontuada em 5 pontos e discordância plena em 1 ponto, refletindo a percepção e compreensão dos respondentes frente às assertivas propostas (Ferreira, 2004).

As médias das respostas aos itens foram desmembradas e classificadas em Zona de Perigo (vermelho) com médias entre 1,00 e 2,33 pontos e apontou para uma percepção negativa, indicando a necessidade de mudanças urgentes; Zona de Alerta (amarelo) com médias entre 2,34 e 3,67 pontos e indicou dificuldades que 
demandavam mudanças sem o caráter de urgência e, por último, Zona de Conforto (verde) com médias entre 3,68 e 5,00 pontos e indicou uma percepção positiva, denotando êxito naquilo que estava sendo pesquisado (Ferreira, 2004; Moraes et al., 2012; Ferreira et al., 2013; Perego; Batista, 2016).

\section{RESULTADOS E DISCUSSÃO}

A análise estatística consistiu em descrever as variáveis de interesse, para o que foram construídas tabelas de frequências, porcentagens e médias relacionando as informações coletadas com os profissionais da área da saúde. Estes elementos forneceram as informações necessárias para traçar um perfil dos participantes.

A variável "sexo" demonstrou que $84,9 \%$ (208) dos profissionais de saúde são do sexo feminino e $15,1 \%$ (37) são do sexo masculino.

O maior número de profissionais do sexo feminino é uma característica que também foi observada por Pinto, Menezes e Villa (2010) em estudo na ESF. Entretanto, destaca-se que esta diferença não necessariamente interfere na avaliação das atitudes colaborativas observadas em uma equipe interprofissional, mas sim a atuação de cada profissional dentro da equipe em que é integrante (Abed, 2015).

Em relação a variável "titulação", apenas 17,1\% ( $N=42)$ possuem apenas a graduação. Possuem especialização 25,7\% ( $N=63)$; mestrado foi declarado por $27,3 \%(\mathrm{~N}=67) ; 15,1 \%(\mathrm{~N}=37)$ possuem doutorado; $2,9 \% \quad(\mathrm{~N}=7)$ possuem pósdoutorado; e 11,8\% ( $\mathrm{N}=29)$ apresentam outros cursos.

Abed (2015) comenta que uma porcentagem elevada de profissionais de nível superior com formação específica para o nível em que atua, pode garantir equipes com maior prática colaborativa e maior colaboração interprofissional.

Em relação à "área de atuação", 45,7\% (N=112) atuam nos serviços de saúde como profissional da assistência e prática docente; $26,5 \%(\mathrm{~N}=65)$ atuam em equipes da Estratégia de Saúde da Família (ESF); 18,4\% (N=45) atuam na Atenção Básica à Saúde; 1,6\% (N=4) atuam nas equipes do Núcleo de Apoio à Saúde da 
Família (NASF); 5,3\% (N=13) atuam na Saúde do Trabalhador; e 2,4\% (N=6) atuam na área da Saúde Mental.

Ellery (2014) considera existir obstáculo para a construção de projetos interprofissionais na ESF e identifica condições e possibilidades que favoreçam a interprofissionalidade, como: dimensão subjetiva, coletiva e organizacional. A dimensão subjetiva envolve a identificação com o modelo assistencial da ESF, saber lidar com as frustações e afetividade. A dimensão coletiva envolve a pactuação de um projeto em comum, engajamento mútuo e repertórios compartilhados. A dimensão organizacional, significa pensar que as unidades de saúde são espaços potenciais de ensino, pesquisa e assistência.

$\mathrm{Na}$ variável "tempo de trabalho com sua equipe atual", 45,7\% dos profissionais $(\mathrm{N}=112)$ estão vinculados à atual equipe de trabalho por período igual ou inferior a 4 anos; 32,2\% dos profissionais ( $N=79$ ) estão entre 5 e 10 anos; 9,8\% $(\mathrm{N}=24)$ estão entre 11 e 14 anos; $5,7(\mathrm{~N}=14)$ estão entre 15 e 19 anos; 2,9\% $(\mathrm{N}=7)$ estão entre 20 e 24 anos; 2,0\% (N=5) estão entre 25 e 30 anos; e 1,6\% (N=4) dos profissionais estão vinculados à atual equipe 30 anos ou mais.

Badlwin (2012) aponta que as relações interpessoais em equipes de trabalho, a depender do tempo de trabalho na mesma equipe, possibilitam a construção da confiança, do respeito e da abertura da comunicação com o outro. As relações interpessoais têm a capacidade de criar vínculos, sendo estes os determinantes de qualidade da colaboração.

Segundo Arruda e Moreira (2018), o nível de interação entre profissionais, está fortemente relacionado com o estreitamento das relações interpessoais e abertura de canais de comunicação durante a dinâmica do processo de trabalho.

A Figura 1 apresenta a média geral dos 23 itens da escala AITCS II-BR para a amostra de 245 respondentes. Os itens foram classificados em zona de conforto, alerta e perigo (Perego, 2015; Perego e Batista, 2016). 


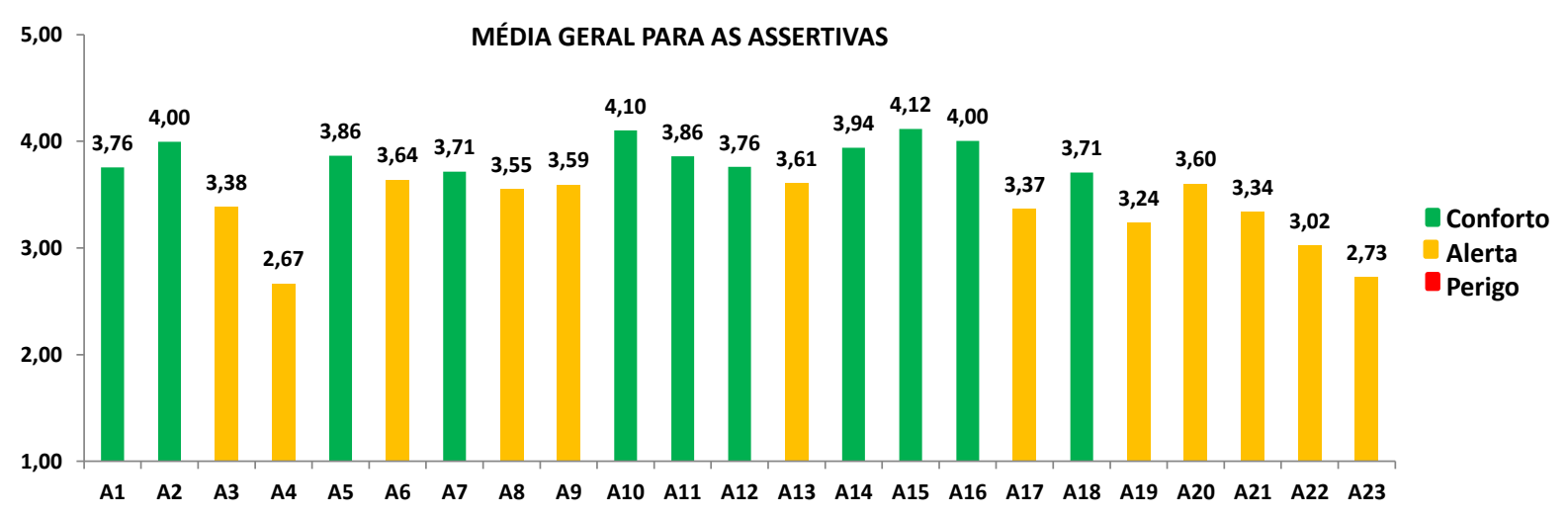

Figura 1. Média geral dos 23 itens da escala AITCS II-BR classificadas em zona de conforto, alerta e perigo, UNIFESP, 2019, N=245.

Com relação a classificação de Perego (2015) e Perego e Batista (2016) para as assertivas, em Zona de conforto, Zona de alerta e Zona de Perigo, verificou-se que na média geral 12 assertivas encontram-se em Zona de Alerta, o que indica a necessidade de aprimoramento e 11 assertivas em Zona de Conforto, o que indica que os aspectos avaliados estão presentes na população investigada e requerem manutenção.

Os dados da Figura 1 serão apresentados separadamente com ênfase nas assertivas de cada Dimensão (Parceria, Cooperação e Coordenação).

Todos os 23 itens da escala AITCS II-BR complementam a frase de contextualização "Quando estamos trabalhando em equipe, os profissionais da minha equipe...", e o participante assina o nível de concordância ou discordância para cada um dos itens.

O Quadro 1 apresenta os itens da Dimensão Parceria. 
Quadro 1. Itens da Dimensão Parceria do AITCS II-BR (BISPO e ROSSIT, 2018).

\begin{tabular}{|c|c|}
\hline \multicolumn{2}{|r|}{ Quando estamos trabalhando em equipe, os profissionais da minha equipe... } \\
\hline 1 & $\begin{array}{l}\text {... incluem os pacientes na definição de metas relacionadas ao cuidado que } \\
\text { receberão. }\end{array}$ \\
\hline 2 & $\begin{array}{l}\ldots \text { ouvem os desejos dos pacientes quando determinam o processo de } \\
\text { cuidado escolhido pela equipe. }\end{array}$ \\
\hline 3. & ... encontram-se regularmente para discutir o cuidado ao paciente. \\
\hline 4. & $\begin{array}{l}\text {.. coordenam serviços de saúde e sociais (por exemplo: finanças, trabalho, } \\
\text { moradia, relações com a comunidade, serviços espirituais) com base nas } \\
\text { necessidades de cuidado do paciente. }\end{array}$ \\
\hline 5. & $\begin{array}{l}\text {... utilizam comunicação coesa e coerente para discutir o cuidado ao } \\
\text { paciente. }\end{array}$ \\
\hline 6. & ... estão envolvidos na definição de metas para cada paciente. \\
\hline 7. & $\begin{array}{l}\text {... incentivam uns aos outros, os pacientes e seus familiares para utilizar os } \\
\text { conhecimentos e as habilidades que cada um pode trazer para desenvolver } \\
\text { planos de cuidado. }\end{array}$ \\
\hline 8. & ... trabalham com o paciente e sua família no ajuste dos planos de cuidado. \\
\hline
\end{tabular}

A Tabela 1 apresenta as respostas obtidas na Dimensão Parceria, com oito itens, da AITCS II-BR aplicada com os profissionais de saúde.

Tabela 1. Distribuição das respostas obtidas na Dimensão Parceria, UNIFESP, 2019 $(\mathrm{N}=245)$.

\begin{tabular}{|c|c|c|c|c|c|c|c|c|c|c|c|c|c|c|c|c|c|}
\hline \multicolumn{18}{|c|}{ PARCERIA } \\
\hline & \multicolumn{2}{|c|}{ A1 } & \multicolumn{2}{|c|}{ A2 } & \multicolumn{2}{|c|}{ A3 } & \multicolumn{2}{|c|}{ A4 } & \multicolumn{2}{|c|}{ A5 } & \multicolumn{2}{|c|}{ A6 } & \multicolumn{2}{|c|}{ A7 } & \multicolumn{2}{|c|}{ A8 } & \multirow[b]{2}{*}{ média } \\
\hline & $\mathrm{N}$ & $\%$ & $\mathrm{~N}$ & $\%$ & $\mathrm{~N}$ & $\%$ & $\mathrm{~N}$ & $\%$ & $\mathrm{~N}$ & $\%$ & $\mathrm{~N}$ & $\%$ & $\mathrm{~N}$ & $\%$ & $\mathrm{~N}$ & $\%$ & \\
\hline Nunca & 15 & 6,1 & 8 & 3,3 & 19 & 7,8 & 68 & 27,8 & 9 & 3,7 & 19 & 7,8 & 14 & 5,7 & 15 & 6,1 & 8,5 \\
\hline Raramente & 23 & 9,4 & 13 & 5,3 & 43 & 17,6 & 42 & 17,1 & 20 & 8,2 & 20 & 8,2 & 23 & 9,4 & 45 & 18,4 & 11,7 \\
\hline Às vezes & 53 & 21,6 & 45 & 18,4 & 66 & 26,9 & 59 & 24,1 & 47 & 19,2 & 59 & 24,1 & 58 & 23,7 & 43 & 17,6 & 22 \\
\hline Frequentemente & 72 & 29,4 & 88 & 35,9 & 62 & 25,3 & 54 & 22,0 & 91 & 37,1 & 81 & 33,1 & 75 & 30,6 & 75 & 30,6 & 30,5 \\
\hline Sempre & 82 & 33,5 & 91 & 37,1 & 55 & 22,4 & 22 & 9,0 & 78 & 31,8 & 66 & 26,9 & 75 & 30,6 & 67 & 27,3 & 27,3 \\
\hline Total & 245 & 100,0 & 245 & 100,0 & 245 & 100,0 & 245 & 100,0 & 245 & 100,0 & 245 & 100,0 & 245 & 100,0 & 245 & 100,0 & 100,0 \\
\hline
\end{tabular}

Em relação à Dimensão Parceria, os itens A3 e A4 estão em Zona de alerta. Estes dois itens demonstram a necessidade de aprimoramento no que diz respeito, principalmente, ao diálogo sobre o paciente e sobre a integralidade da atenção. No item $A 3,26,9 \%(N=66)$ responderam "às vezes" e 25,3\% $(N=62)$ responderam "Frequentemente". No item $\mathrm{A} 4,27,8 \%$ dos participantes $(\mathrm{N}=68)$ responderam que "nunca" e $24,1 \%(N=59)$ responderam que "às vezes". 
Na Tabela 1, observa-se que os itens A3 e A8 apresentam maior distribuição entre as opções de respostas. Isso indica que os conteúdos abordados precisam estar mais claros no trabalho em equipe, principalmente no que se refere à parceria. Também no item A4, observa-se alta porcentagem de respostas na opção "nunca" e a tendência centrante com escolha da opção "às vezes", o que pode indicar incerteza ou falta de definição em relação ao iten.

O trabalho em equipe envolve profissionais de diferentes áreas da saúde que compartilham uma identidade de equipe, que têm clareza dos papéis de cada membro, dos objetivos traçados e das atividades a serem realizadas em equipe e, também, que trabalhem de forma integrada e em mútua interdependência em todas as atividades necessárias (Reeves; Xyrichis; Zwarenstein, 2018).

Para Orchard et al. (2012), a parceria é essencial dentro da prática colaborativa, principalmente porque reconhece a importância da participação dos pacientes e familiares como protagonistas do cuidado em saúde, além da tomada de decisão coletiva e do planejamento integrado, colaborando para o trabalho em equipe interprofissional.

Para o trabalho em equipe interprofissional deve-se considerar os níveis de Parceria, Cooperação e Coordenação. Estas dimensões são especificadas no AITCS II-BR (BISPO; ROSSIT, 2018) e caracterizam as relações entre profissões na prestação de cuidados centrados no paciente/usuário (HPAC, 2019).

A Figura 2 apresenta a média de cada item e a média geral da Dimensão Parceria. 


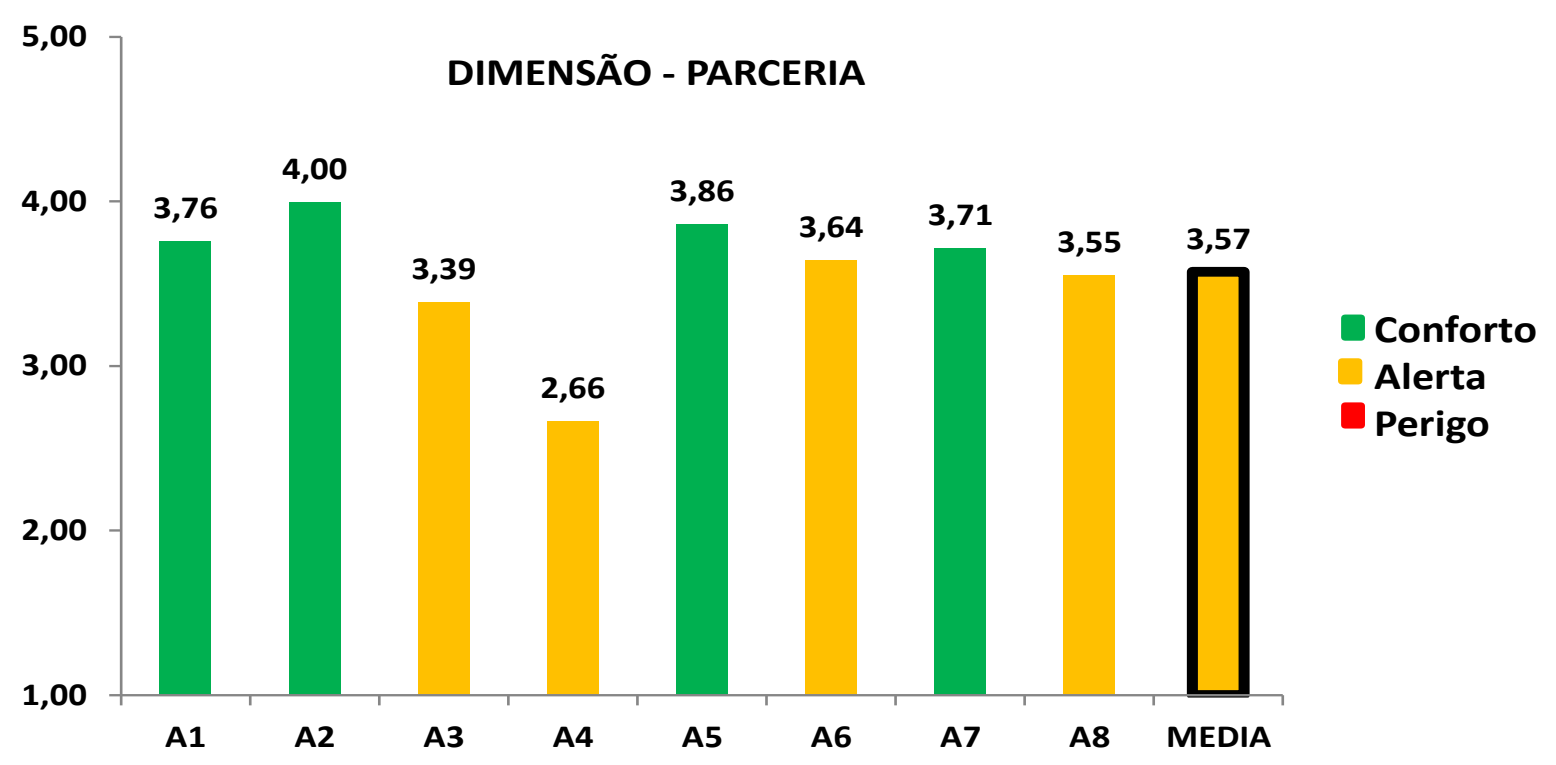

Figura 2. Média de cada item e a média geral da Dimensão Parceria da AITCS IIBR, UNIFESP, 2019, N=245.

No item $A 1,33,5 \%(N=82)$ dos profissionais responderam "sempre" e $29,4 \%$ $(\mathrm{N}=72)$ responderam "frequentemente" frente à inclusão dos pacientes na definição de metas. No $A 2,37,1 \%(\mathrm{~N}=91)$ responderam que "sempre" e $35,9 \% \quad(\mathrm{~N}=88)$, responderam "frequentemente" em relação a ouvir os desejos dos pacientes quando determinam o processo de cuidado. No $A 3,26,9 \%(\mathrm{~N}=66)$ responderam "às vezes" e 25,3\% ( $N=62)$ responderam "Frequentemente" em relação à encontrar-se regularmente para discutir o cuidado ao paciente. No $A 4,27,8 \%(\mathrm{~N}=68)$ dos profissionais responderam "nunca" e $24,1 \%(\mathrm{~N}=59)$ responderam "às vezes" em relação à coordenar serviços de saúde e sociais com base nas necessidades de cuidado. No $A 5$, da dimensão Parceria, 37,1\% (N=91) responderam que "frequentemente", $\quad 31,8 \% \quad(\mathrm{~N}=78)$ responderam "sempre" e $19,2 \% \quad(\mathrm{~N}=47)$ responderam "às vezes". No item $A 6$, da dimensão Parceria, destaca-se que 33,1\% $(\mathrm{N}=81)$ responderam "frequentemente" para esta assertiva e $26,9 \% \quad(\mathrm{~N}=66)$, responderam que "sempre". No $A 7$, referente à dimensão Parceria, 30,6\% ( $\mathrm{N}=75)$ responderam que "frequentemente" e a mesma proporção $(30,6 \%)$ respondeu "sempre". No A8, da dimensão Parceria, observa-se que 30,6\% ( $\mathrm{N}=75)$ responderam "frequentemente", e, 27,3\% (N=67) responderam "sempre". 
Nota-se que os participantes, no contexto da equipe profissional, fazem a escuta dos desejos dos pacientes ao definir metas e planejar o processo de cuidado, e que, frequentemente, incentivam uns aos outros, os pacientes e seus familiares para utilizar os conhecimentos e as habilidades que cada um pode trazer para desenvolver planos de cuidado. Os profissionais expressam que se encontram, regularmente, para discutir o cuidado ao paciente e que utilizam comunicação coesa e coerente para discutir este cuidado com o paciente. Entretanto, fica evidente a ausência destes profissionais na coordenação dos serviços de saúde e sociais com base nas necessidades de cuidado.

Costa (2016) descreve sobre a importância do estabelecimento dos vínculos profissionais e subsídios para a ampliação da saúde, como essenciais para a prática interprofissional. Para tanto, são necessárias mudanças referentes à comunicação, à socialização dos papéis profissionais e ao processo de trabalho em saúde (OMS, 2010; Gilbert, 2010; Silva et al., 2015).

Desse modo, observa-se que a média geral da Dimensão Parceria está em zona de alerta (A3, A4, A6 e A8). Para Reeves, Xyrichis e Zwarenstein (2018), o trabalho da equipe interprofissional engloba vários elementos centrais, incluindo: identidade de equipe, clareza, interdependência, integração e responsabilidade compartilhada. Segundo este autor, quando esses elementos não estão fortalecidos, o trabalho em equipe necessita ser reavaliado.

De acordo com Orchard et al (2012), as parcerias dentro da prática colaborativa "reconhecem e respeitam o papel e as contribuições dos pacientes e suas famílias, como parceiros em seus cuidados". Relacionamentos de trabalho em parceria e colaboração exige confiança por parte das equipes. A confiança parece estar correlacionada com experiência e competência. Parcerias envolvem "compartilhar responsabilidades entre as partes, tomada de decisão compartilhada e planejamento compartilhado para intervenções".

O Quadro 2 apresenta os itens da Dimensão Cooperação. 
Quadro 2. Itens da Dimensão Cooperação da AITCS II-BR (BISPO e ROSSIT, 2018).

\begin{tabular}{|c|c|}
\hline & do estamos trabalhando em equipe, os profissionais da minha $\epsilon$ \\
\hline 9 & ...compartilham poder uns com os outros. \\
\hline 10 & ...respeitam-se e confiam uns nos outros. \\
\hline 11 & ertos e honestos uns com os outros. \\
\hline 12 & ...refletem sobre sua prática e comr \\
\hline 13 & $\begin{array}{l}\text {...esforçam-se para atingir soluções mutuamente satisfatórias para as } \\
\text { diferenças de opinião. }\end{array}$ \\
\hline 14 & ...entendem os limites do que cada um pode fazer. \\
\hline 15 & $\begin{array}{l}\text {...entendem que existem conhecimentos e habilidades compartilhadas entre } \\
\text { os profissionais de saúde na equipe. }\end{array}$ \\
\hline 16 & timento de confiança entre os membros da equipe. \\
\hline
\end{tabular}

A Tabela 2 apresenta as respostas obtidas na Dimensão Cooperação da AITCS II-BR aplicada com os profissionais de saúde.

Tabela 2. Distribuição das respostas obtidas na Dimensão Cooperação, com oito itens, na amostra estudada, UNIFESP, 2019, N= 245.

\begin{tabular}{|c|c|c|c|c|c|c|c|c|c|c|c|c|c|c|c|c|}
\hline \multicolumn{17}{|c|}{ COOPERAÇÃO } \\
\hline & \multicolumn{2}{|c|}{ A9 } & \multicolumn{2}{|c|}{ A10 } & \multicolumn{2}{|c|}{ A11 } & \multicolumn{2}{|c|}{ A12 } & \multicolumn{2}{|c|}{ A13 } & \multicolumn{2}{|c|}{ A14 } & \multicolumn{2}{|c|}{ A15 } & \multicolumn{2}{|c|}{ A16 } \\
\hline & $\mathrm{N}$ & $\%$ & $\mathrm{~N}$ & $\%$ & $\mathrm{~N}$ & $\%$ & $\mathrm{~N}$ & $\%$ & $\mathrm{~N}$ & $\%$ & $\mathrm{~N}$ & $\%$ & $\mathrm{~N}$ & $\%$ & $\mathrm{~N}$ & $\%$ \\
\hline Nunca & 18 & 7,3 & 4 & 1,6 & 6 & 2,4 & 6 & 2,4 & 20 & 8,2 & 10 & 4,1 & 5 & 2,0 & 5 & 2,0 \\
\hline Raramente & 24 & 9,8 & 11 & 4,5 & 17 & 6,9 & 28 & 11,4 & 26 & 10,6 & 16 & 6,5 & 10 & 4,1 & 19 & 7,8 \\
\hline Às vezes & 65 & 26,5 & 38 & 15,5 & 62 & 25,3 & 58 & 23,7 & 49 & 20,0 & 49 & 20,0 & 40 & 16,3 & 47 & 19,2 \\
\hline Frequentemente & 73 & 29,8 & 99 & 40,4 & 84 & 34,3 & 81 & 33,1 & 87 & 35,5 & 77 & 31,4 & 89 & 36,3 & 75 & 30,6 \\
\hline Sempre & 65 & 26,5 & 93 & 38,0 & 76 & 31,0 & 72 & 29,4 & 63 & 25,7 & 93 & 38,0 & 101 & 41,2 & 99 & 40,4 \\
\hline Total & 245 & 100,0 & 245 & 100,0 & 245 & 100,0 & 245 & 100,0 & 245 & 100,0 & 245 & 100,0 & 245 & 100,0 & 245 & 100,0 \\
\hline
\end{tabular}

Em relação à Dimensão Cooperação, alerta-se para os itens A9 e A13 estão em Zona de alerta. Estes dois itens apontam para a necessidade de aprimoramento no que diz respeito ao trabalho em equipe e as competências para uma prática colaborativa.

Para Orchard et al (2012), a cooperação é essencial no trabalho em equipe, pois permite uma mudança de postura e proporciona uma experiência coletiva contrária à comunicação distorcida e aos conflitos de papéis, possibilitando a colaboração. Esta colaboração compreende o compartilhamento, parceria, 
interdependência, poder e envolve a reflexão e ação mútua em busca de respostas às necessidades de saúde dos usuários (Reeves; Xyrichis; Zwarenstein, 2018).

No item $A 9,29,8 \%(\mathrm{~N}=73)$ responderam "Frequentemente", 26,5\% (N=65) responderam "às vezes" e na mesma proporção $(26,5 \%, N=65)$ responderam "sempre". No item A13, 35,5\% (N=87) responderam "Frequentemente" e 25,7\% (N63) responderam "sempre".

Os itens A10, A15 e A16 apresentam mais de 70\% das respostas "frequentemente" e "sempre", o que demonstra contradição com os itens A9 e A13. Quando o trabalho em equipe é limitado, existe competitividade entre os membros da equipe, a comunicação é distorcida e os conflitos de papéis podem impedir a cooperação (ORCHARD et al., 2012, p. 60).

A Figura 3 apresenta a média geral de cada assertiva e a média geral da Dimensão Cooperação.

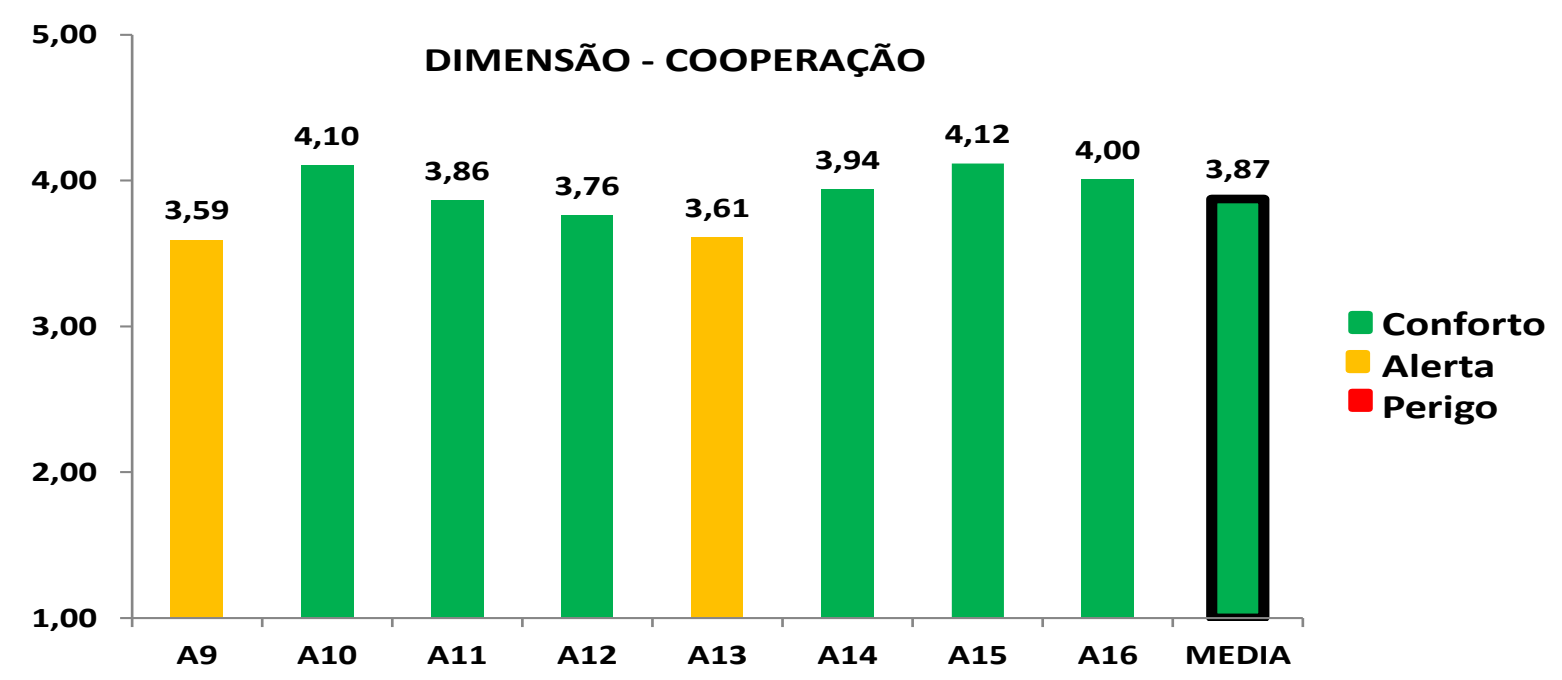

Figura 3. Média de cada item e a média geral da Dimensão Cooperação da AITCS II-BR, UNIFESP, 2019, N= 245.

No item A9, da Dimensão Cooperação, 29,8\% (N=73) responderam "frequentemente", 26,5\% ( $\mathrm{N}=65)$ responderam "às vezes" e na mesma proporção de 26,5\% ( $N=65)$ responderam "sempre". No item $A 10,40,4 \%(N=99)$ responderam "frequentemente" e 38,0\% (N=93) responderam "sempre". No item $A 11,34,3 \%$ $(\mathrm{N}=84)$ responderam "frequentemente", enquanto que $31,0 \%(\mathrm{~N}=76)$ responderam 
"sempre" e 25,3\% (N=62) responderam "às vezes". No $A 12,33,1 \%(\mathrm{~N}=81)$ responderam "frequentemente" e 29,4\% ( $N=72)$ responderam "sempre". No A13, $35,5 \%$ ( $N=87$ profissionais) responderam "frequentemente" e 25,7\% (N=63) responderam "sempre". No A14, 38,0\% (93 participantes) responderam "sempre" e $31,4 \%(\mathrm{~N}=77)$ responderam "frequentemente". No $\mathrm{A} 15,41,2 \%(\mathrm{~N}=101)$ responderam "sempre" para esta assertiva e 36,3\% (N=89) responderam "frequentemente". E no item A16 desta Dimensão, 40,4\% (N=99) responderam "sempre" para esta assertiva e 30,6\% (N=75) responderam "frequentemente".

Nota-se uma percepção positiva dos participantes de saúde em relação à compartilhar poder uns com os outros, respeitar-se e confiar uns nos outros, como também, manifestam serem abertos e honestos uns com os outros, refletem sobre sua prática e compartilham suas percepções. Esforçam-se para atingir soluções mutuamente satisfatórias para as diferenças de opiniões. Sempre entendem os limites que cada um pode fazer e entendem que existem conhecimentos e habilidades compartilhadas entre os profissionais de saúde e da equipe. Para que a cooperação aconteça é necessário, também, a tomada de decisão compartilhada.

O Quadro 3 apresenta os itens da Dimensão Coordenação.

Quadro 3. Itens da Dimensão Coordenação da AITCS II-BR (Bispo e Rossit, 2018).

\begin{tabular}{|c|l|}
\hline \multicolumn{2}{|c|}{ Quando estamos trabalhando em equipe, os profissionais da minha equipe... } \\
\hline 17 & $\begin{array}{l}\text {...aplicam uma definição única de Prática colaborativa interprofissional } \\
\text { no cenário da prática. }\end{array}$ \\
\hline 18 & ...distribuem igualmente as metas acordadas entre os membros da equipe. \\
\hline 19 & $\begin{array}{l}\text {...incentivam e apoiam a comunicação aberta, incluindo pacientes e seus } \\
\text { familiares nas reuniões da equipe. }\end{array}$ \\
\hline 20 & ...utilizam um processo de acordo para resolver conflitos. \\
\hline 21 & $\begin{array}{l}\text {...defendem que o líder da equipe varie dependendo das necessidades dos } \\
\text { pacientes. }\end{array}$ \\
\hline 22 & ...escolhem juntos o líder para a equipe. \\
\hline 23 & ....apoiam abertamente a inclusão do paciente nas reuniões da equipe. \\
\hline
\end{tabular}

A Tabela 3 apresenta as respostas obtidas na Dimensão Coordenação da AITCS II-BR aplicada com os profissionais de saúde. 
Tabela 3. Distribuição das respostas obtidas na Dimensão Coordenação, com sete itens, na amostra estudada, UNIFESP, 2019, N= 245.

\begin{tabular}{|c|c|c|c|c|c|c|c|c|c|c|c|c|c|c|}
\hline \multicolumn{15}{|c|}{ COORDENAÇÃO } \\
\hline & \multicolumn{2}{|c|}{ A17 } & \multicolumn{2}{|c|}{ A18 } & \multicolumn{2}{|c|}{ A19 } & \multicolumn{2}{|c|}{ A20 } & \multicolumn{2}{|c|}{ A21 } & \multicolumn{2}{|c|}{ A22 } & \multicolumn{2}{|c|}{ A23 } \\
\hline & $\mathrm{N}$ & $\%$ & $\mathrm{~N}$ & $\%$ & $\mathrm{~N}$ & $\%$ & $\mathrm{~N}$ & $\%$ & $\mathrm{~N}$ & $\%$ & $\mathrm{~N}$ & $\%$ & $\mathrm{~N}$ & $\%$ \\
\hline Nunca & 15 & 6,1 & 9 & 3,7 & 32 & 13,1 & 14 & 5,7 & 35 & 14,3 & 61 & 24,9 & 64 & 26,1 \\
\hline Raramente & 44 & 18,0 & 17 & 6,9 & 42 & 17,1 & 28 & 11,4 & 31 & 12,7 & 31 & 12,7 & 50 & 20,4 \\
\hline Às vezes & 70 & 28,6 & 74 & 30,2 & 56 & 22,9 & 65 & 26,5 & 55 & 22,4 & 50 & 20,4 & 52 & 21,2 \\
\hline Frequentemente & 71 & 29,0 & 86 & 35,1 & 66 & 26,9 & 74 & 30,2 & 64 & 26,1 & 46 & 18,8 & 47 & 19,2 \\
\hline Sempre & 45 & 18,4 & 59 & 24,1 & 49 & 20,0 & 64 & 26,1 & 60 & 24,5 & 57 & 23,3 & 32 & 13,1 \\
\hline Total & 245 & 100,0 & 245 & 100,0 & 245 & 100,0 & 245 & 100,0 & 245 & 100,0 & 245 & 100,0 & 245 & 100,0 \\
\hline
\end{tabular}

Observa-se na Tabela 3 que apenas o item A18 encontra-se em Zona de conforto, indicando êxito em relação à coordenação do trabalho em equipe. Os demais itens estão em Zona de Alerta, o que indica uma posição indefinida e uma necessidade de aprimoramento em relação à coordenação do cuidado. A Dimensão Coordenação, segundo Orchard et al. (2012), envolve planejamento e é essencial para uma relação de trabalho eficaz. A comunicação e a ajuda mútua são prioritárias para considerar esta Dimensão.

No item $A 19,20,0 \% \quad(N=49)$ responderam "sempre", $17,1 \% \quad(N=42)$ responderam "raramente" e $13,1 \%(\mathrm{~N}=32)$ responderam "nunca". No item $\mathrm{A} 22$, 24,9\% ( $N=61$ ) responderam que "nunca" escolhem juntos o líder da equipe, e 23,3\% $(\mathrm{N}=57)$ responderam "sempre". No item $\mathrm{A} 23,26,1 \%(\mathrm{~N}=64)$ responderam "nunca", $21,2 \%(N=52)$ responderam "às vezes" e, 20,4\% $(N=50)$ responderam "raramente".

Segundo Reeves, Xyrichis e Zwarenstein (2018), para que a coordenação aconteça no trabalho em equipe é necessária uma identidade compartilhada. Os itens A22 e A23 demonstram que esta identidade compartilhada está enfraquecida.

Dessa maneira, uma série de mecanismos aperfeiçoa a prática colaborativa, incluindo: práticas gerenciais de apoio; identificação e apoio aos líderes; decisão de mudar a cultura e as atitudes dos profissionais de saúde; vontade de atualizar; e uma legislação adequada que elimine as barreiras para o trabalho colaborativo (Anderson, Ford, Kinnair, 2016 ; OMS, 2010; CIHC, 2010).

A Figura 4 apresenta a média de cada item e a média geral da Dimensão Coordenação. 


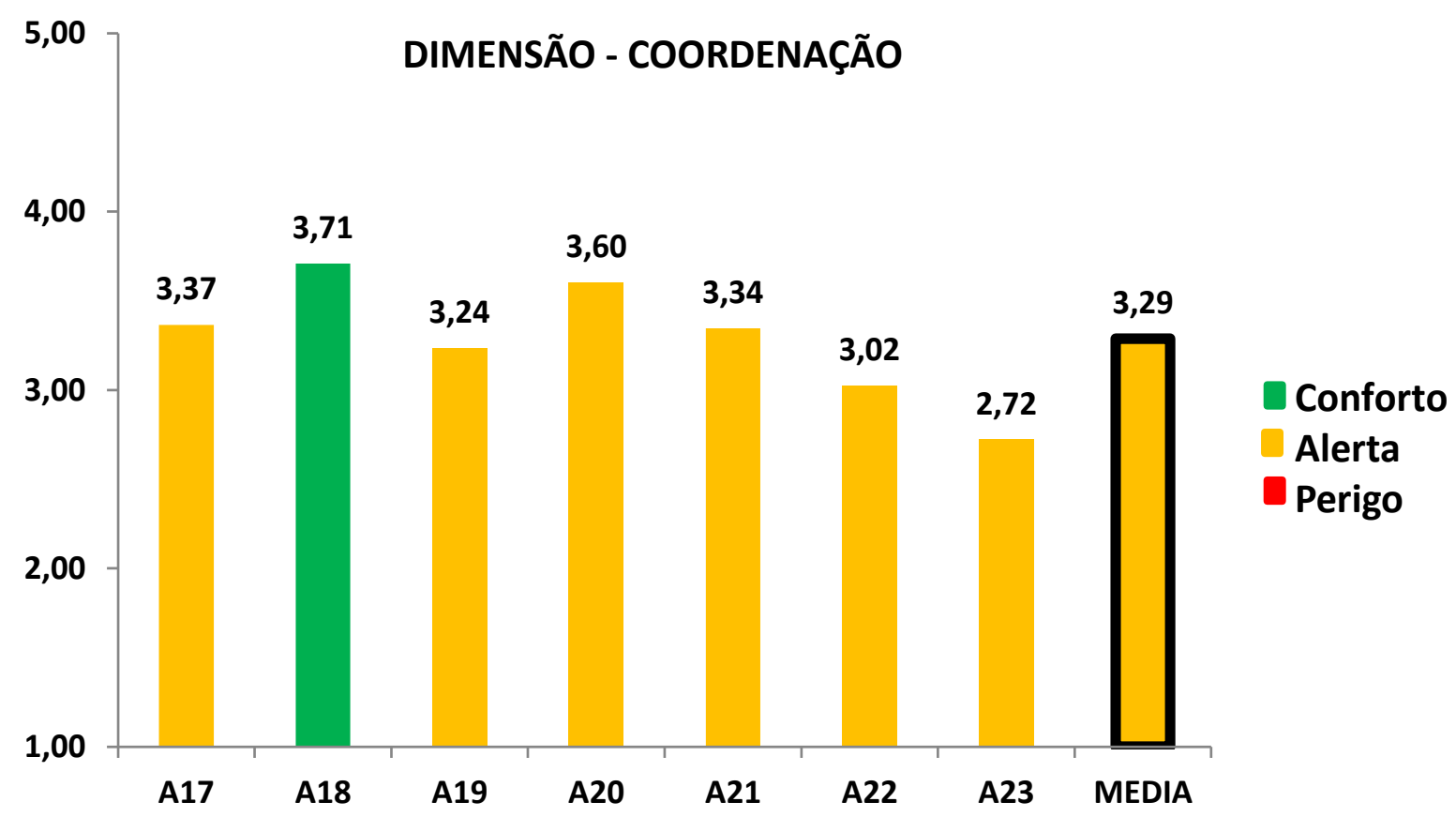

Figura 4. Média de cada item e média geral da Dimensão Coordenação da AITCS IIBR, UNIFESP, 2019, N= 245.

No item $A 17$, da Dimensão Coordenação, 29,0\% ( $\mathrm{N}=71)$ responderam "frequentemente" e 28,6\% ( $N=70)$ responderam "às vezes". No item $A 18,35,1 \%$ $(\mathrm{N}=86)$ responderam "frequentemente", $30,2 \%(\mathrm{~N}=74)$ "às vezes" e $24,1 \%(\mathrm{~N}=59)$ "sempre". No $A 19,26,9 \%(\mathrm{~N}=66)$ responderam "frequentemente", 22,9\% $(\mathrm{N}=56)$ "às vezes", $20,0 \% \quad(\mathrm{~N}=49)$ "sempre", $17,1 \% \quad(\mathrm{~N}=42)$ "raramente" e $13,1 \% \quad(\mathrm{~N}=32)$ responderam "nunca". No item A20, observa-se que $30,2 \%(\mathrm{~N}=74)$ responderam "frequentemente", 26,5\% ( $\mathrm{N}=65)$ "às vezes" e $26,1 \%(\mathrm{~N}=64)$ "sempre". No $A 21$, $26,1 \%(\mathrm{~N}=64)$ responderam "frequentemente" e $24,5 \%(\mathrm{~N}=60)$ "sempre". No item A22, 24,9\% ( $N=61)$ responderam que "nunca" escolhem juntos o líder da equipe, e $23,3 \%(\mathrm{~N}=57)$ responderam "sempre" para este item. No $A 23,26,1 \%(\mathrm{~N}=64)$ responderam "nunca", $21,2 \%(N=52)$ responderam "às vezes" e $20,4 \%(N=50)$ responderam "raramente".

Nota-se que os participantes "frequentemente" aplicam uma definição única de Prática Colaborativa Interprofissional no cenário de prática; distribuem igualmente as metas acordadas entre os membros da equipe; incentivam e apoiam a comunicação aberta, incluindo pacientes e seus familiares nas reuniões da equipe; 
utilizam o processo de resolução de problemas para resolver conflitos; como também, defendem que o líder da equipe varie dependendo das necessidades dos pacientes. Manifestam que "nunca" escolhem juntos o líder da equipe, e também, "nunca" apoiam abertamente a inclusão do paciente nas reuniões da equipe.

De acordo com Orchard et al (2012), a coordenação é um processo que resulta no uso eficiente de tempo, esforço e recursos; padronização de procedimentos (levando a resultados de qualidade); tempos de resposta rápida; e uma boa reputação. A coordenação envolve uma série de interligações de atividades de planejamento de cuidado criadas com e para a equipe. eficaz é essencial para a colaboração e depende de relações coletivas de trabalho eficazes entre serviços e sistemas interorganizacionais que fornecem tempo e recursos necessários envolvendo pacientes e suas famílias.

Para as tarefas coletivas, a prática interprofissional proporciona uma melhor qualidade na saúde ao favorecer habilidades necessárias para o desenvolvimento destas tarefas (Rocha et al., 2016; Reeves, 2016; Dias et al., 2016).

Considerando as três dimensões (Parceria, Cooperação e Coordenação) da escala AITCS II-BR (Bispo e Rossit, 2018) as seguintes competências colaborativas são apontadas como essenciais para 0 trabalho em equipe interprofissional: comunicação interprofissional; cuidado/atenção ao paciente/usuário; esclarecimento de funções/ clarificação de papeis; funcionamento da equipe; liderança colaborativa; resolução de conflitos interprofissionais; valores éticos para a prática interprofissional; responsabilidades e trabalho em equipe (HPAC, 2019; CIHC, 2010; IPEC, 2011).

\section{CONSIDERAÇÕES FINAIS}

Este estudo analisou o nível de colaboração interprofissional entre os profissionais de saúde da região Nordeste do Brasil, a partir da escala AITCS II-BR ${ }^{8}$ traduzida, adaptada e validada para o contexto brasileiro. 
Indica-se, assim, a necessidade de novos estudos para maior aprofundamento dessa temática e ampliação do repertório de competências essenciais para a colaboração interprofissional. Outro desafio será realizar estudos longitudinais de melhorias do trabalho em equipe e da colaboração interprofissional, o que poderá fornecer informações valorosas sobre o modo como uma equipe trabalha e atua nos contextos de atenção à saúde e subsidiar o desenvolvimento de ações de educação permanente para o fortalecimento da força de trabalho em saúde para maior resolutividade das demandas existentes nas realidades brasileiras.

\section{REFERÊNCIAS BIBLIOGRÁFICAS}

ABED, M.M. Adaptação e Validação da versão brasileira da escala Jefferson de atitudes relacionadas à colaboração interprofissional: Um estudo em profissionais da Atenção Básica [manuscrito]. Dissertação de Mestrado pela Universidade Federal de Goiás, Faculdade de Medicina, Programa de Pós-Graduação em Ensino na Saúde (Profissional), Goiânia, 2015.

ANDERSON, E.S; FORD, J.J; KINNAIR, D.D.J. Interprofessional Education and Practice Guide. Developing practice-based interprofessional learning using a short placement model. of Interprofessional. Journal of Interprofessional Care. n.6: 2016.

ARRUDA, L.S, MOREIRA, C.O.F. Interprofessional collaboration: a case study regarding the professionals of the Care Center for Elderly, Rio de Janeiro State University (NAI/UERJ), Brazil. Interface (Botucatu), v. 22, n. 64, p. 199-210: 2018.

BALDWIN, A. Exploring the interacional determinants of collaboration on interprofessional practice in community-based geriatric care [tese]. Winnipeg: Faculty of Education, University of Manitoba: 2012.

BARR, H.; KOPPEL, L.; REEVES, S.; HAMMICK, M.; FREETH, D. Effective interprofessional education: argument, assumption, evidence. Oxford: Blackwell Publishing: 2005.

BISPO, E.P.F; ROSSIT, R.A.S. Adaptação transcultural e validação estatística do Assessment of Interprofessional Team Collaboration Scale II. Revista Científica Ágape. v1, 1ª̊edição: 2018.

BODENHEIMER, T., \& Sinsky, C. From Triple to Quadruple Aim: Care of the patient. Annals of Family Medicine. v. 12; n. 6; p. 573-576: 2014. https://doi. org/10.1370/afm.1713.

CIHC. A National Interprofessional Competency Framework. Vancouver: CIHC: 2010.

COSTA, M.V. A educação interprofissional no contexto brasileiro: algumas reflexões. Interface Comunicação Saúde Educação. v. 20, n.56, p. 197-8: 2016.

DIAS, M. S.A. et al. Colaboração interprofissional no Projeto Saúde e Prevenção na Escola. Ciência \& Saúde Coletiva. v.1, n. 6, p. 1789-1798: 2016.

ELLERY, A.E.L. Interprofissionalidade na Estratégia Saúde da Família: condições de possibilidade para a integração de saberes e a colaboração interprofissional, Rev. Interface. v.18, n. 48, p.213-5: 2014. 
FEELEY, D. The Triple Aim or the Quadruple Aim? Four points to help set your strategy. 2017. Retrieved from http://www.ihi.org/communities/blogs/ the-triple-aim-or-the-quadruple-aim-fourpoints-to-help-set-your-strategy.

FERREIRA, B.J. Inovações na formação médica: reflexos na organização do trabalho pedagógico (Tese doutorado). Campinas: São Paulo: 2004.

FERREIRA, B.J.; BATISTA, S.H.; BATISTA, N.A. O Processo de ensino/aprendizagem no mestrado profissional - MP-Norte: análise de uma experiência. IX Congreso Internacional sobre Investigaciónen Didáctica de las Ciencias. Girona. septiembre 9-12. p 1246-1251: 2013.

FRENK, J. et al. Health professionals for a new century: transforming education to strengthen health systems in an interdependent world. Lancet, London, v. 376 n. 9756, p. 1923-1958: 2010.

GILBERT, J.H.V. A National Interprofessional Competency Framework. Saúde Interprofissional Canadense Colaborativo (ClHC): 2010.

HPAC. HEALTH PROFESSIONS ACCREDITORS COLLABORATIVE. Guidance on developing quality interprofessional education for the health professions. Chicago, IL: Health Professions Accreditors Collaborative: 2019.

INTERPROFESSIONAL EDUCATIONAL COLLABORATIVE. Core competencie for interprofessional collaborative practice: 2016 Update. Washington, DC, USA: Interprofessional Education Collaborative. Retrieved from https://aamcmeded.global.ssl.fastly.net/production/media/filer_public/70/9f/709fedd7-3c53-492cb9f0.

IPEC. INTERPROFESSIONAL EDUCATION COLLABORATIVE GROUP. Interprofessional Education Collaborative Expert Panel. Core competencies for interprofessional practice: report on an expert panel. Washington: 2016.

KHALILI, H., Gilbert, J., Lising, D., MacMillan, K., Maxwell, B., Xyrichis, A. Proposta de léxico para o campo interprofi ssional. Publicação conjunta do

InterprofessionalResearch.Global e da Interprofessional.Global: 2019. Disponível em www.research.interprofessional.global.

MORAES, S.G.; JUSTINO, M.L.; JANSEN, B.F.; BARBOSA, E.P.; BRUNO, L.F.C.; PEREIRA, L.A.V. Development and validation of strategy to assess teacching methods in undergraduate disciplines. In: Progress in Education. v. 28. Nova Science Publishers. 2012.

OMS. ORGANIZAÇÃO MUNDIAL DE SAÚDE. Marco para Ação em Educação Interprofissional e Prática Colaborativa. p.1-64: 2010.

ORCHARD, CA. Assessment of Interprofessional Team Collaboration Scale II (AITCS-II). Revised version November: 2015.

ORCHARD, CA; KING, GA; KHALILI, H. Assessment of Interprofessional Team Collaboration Scale (AITCS): Development and Testing of the Instrument. journal of continuing education in the health professions. v.32, n.1, p. 58-67: 2012.

PEDUZZI, M., Norman, I., Coster, S., Meireles, E. Adaptação transcultural e validação da Readiness for Interprofessional Learning Scale no Brasil. Rev Esc Enferm USP, v. 49, pp. 7-15: 2015.

PEREGO, M.G. Aprendizagens compartilhadas na Residência Multiprofissional em saúde. [Dissertação]. Mestrado em Ciência da Saúde. Universidade Federal de São Paulo: 2015. 
PEREGO, M.G.; BATISTA, N.A. Aprendizagens Compartilhadas na Residência Multiprofissional em Saúde. Tempus, Actas de Saúde Colet, v.10, n.4, p.39-51: 2016.

PINTO, E. S. G.; MENEZES, R. M. P. D.; VILLA, T. C. S. Situação de trabalho dos profissionais da Estratégia Saúde da Família em Ceará-Mirim. Revista da Escola da Enfermagem da USP, 44, n. 3, set, p. 657-664: 2010.

PRICE, S., Doucet, S., \& Hall, L. The historical social positioning of nursing and medicine: Implications for career choice, early socialization and interprofessional collaboration. Journal of $\begin{array}{llllllll}\text { Interprofessional } & \text { Care. } & \text { v. 28, } & \text { n. } & 2, & \text { p. } & \text { 103- } & \text { 109: }\end{array}$ https://doi.org/10.3109/13561820.2013.867839.

ROCHA, F. A.A; BARRETO, I.C.H.C; MOREIRA, A.A.E.M.M. Colaboração interprofissional: estudo de caso entre gestores, docentes e profissionais de saúde da família. Interface (Botucatu). v.20, n.57, p. 415-26: 2016.

REEVES, S; XYRICHIS, A; ZWARENSTEIN, M. Teamwork, collaboration, coordination, and networking: Why we need to distinguish between diferente types of interprofessional practice, Journal of Interprofessional Care, v. 32, n.1, p. 1-3: 2018. DOI:10.1080/13561820.2017.1400150.

REEVES, S; LEWIN, S; ESPIN, S; ZWARENSTEIN, M. A conceptual framework for interprofessional teamwork. In: Barr $\mathrm{H}$, editor. Interprofessional teamwork for health and social care. Chichester: Wiley-Blackwell: 2010.

REEVES, S.; Hean, S. Why we need theory to help us better understand the nature of interprofessional education, practice and care. Journal of interprofessional care, Abingdon. v.27, n.1, p. 1-3: 2013.

REEVES, S. et al. A BEME systematic review of the effects of interprofessional education: BEME Guide No. 39. Medical Teacher. V.38, n. 7: 2016.

ROSSIT, R.A.S; FREITAS, M.A.O; BATISTA, S.S.H.S; BATISTA, N.A. Constructing professional identity in Interprofessional Health Education as perceived by graduates. Interface (Botucatu). v.22, Supl.1, p.1399-410: 2018.

SILVA, J. A.M. et al. Educação interprofissional e prática colaborativa na Atenção Primária à Saúde. Rev Esc Enferm USP. v.49, n.2, p. 16-24: 2015.

THIBAULT, G. Reforming health professions education will require culture change and closer ties between classroom and practice. Health Affairs. V. 32; n.11, pp. 1928-1932: 2013. https://doi.org/10.1377/hlthaff.2013.0827. 